\title{
Distorsión relativa de cuatro hidrocoloides irreversibles en modelos de piedra dental tipo IV.
}

\author{
Blas Arles Arauz T. C.D., \\ LEON \\ Arles69@hotmail.com \\ M.Sc. Mercedes Elizabeth López Mena C.D., \\ Práctica Privada \\ Cristian de Jesús Hernández C.D., \\ Práctica Privada \\ Ana Walkiria López Toruño C.D. \\ Práctica Privada.
}

Profesor titular facultad de odontología, Profesor de posgrado Maestría Estética y Rehabilitación Dental UNAN-

Recibido: 15 Septiembre, 2014

Aceptado: Octubre 15, 2014

\section{1- RESUMEN}

El propósito de este estudio fue medir el grado de distorsión de 4 hidrocoloides irreversibles comparando su deformación en el plano (XY). Se diseñó un estudio experimental, efectuado en la facultad de Odontología de la UNAN - LEÓN donde se tomaron las impresiones por cada hidrocoloide seleccionado (Jeltrate, Max Print Cyan, Vival NF, Rite Dent), obteniendo una población de 20 modelos de estudio, 5 por cada marca de hidrocoloide irreversible. La medición de los modelos se realizó en una máquina de coordenadas (X, Y,Z) marca Mitutoyo-Japón que funciona con el programa Geopack 500 con un margen de error de $0.0001 \mathrm{~mm}$. No se presentaron diferencias estadísticamente significativas al aplicar el análisis ANOVA y TUKEY.

Palabras Claves: Diabetes Mellitus, Prevalencia, Factores de Riesgo, Nicaragua

\section{SUMARY}

The intention of this study was to measure the degree of distortion of 4 irreversible hidrocoloid being compared its deformation in the plane (XY). An experimental study was designed, carried out in the faculty of Dentistry of UNAN - LEON where the impressions by each selected hidrocoloid were taken (Jeltrate, Max Print Cyan, Vival NF, Rite Dent), obtaining a population of 20 models of study, 5 by each mark of irreversible hidrocoloid. The measurement of the models was made in a machine of coordinates (X, Y,Z) marks Mitutoyo-Japan that works with the program Geopack 500 with a margin of error of $0.0001 \mathrm{~mm}$. Statistically significant differences when applying did not appear analysis ANOVA and TUKEY.

\section{2- PALABRAS CLAVES}

Distorsión Relativa, Hidrocoloides Irreversibles, Plano XY. 


\section{3- INTRODUCCIÓN}

Los materiales de impresión han sido utilizados desde hace mucho tiempo en la práctica odontológica. Uno de los primeros materiales de impresión fueron los yesos, tiempo después el químico inglés E. C. C. Stanford, por digestión de frondas de ciertas algas pardas con carbonato sódico, obtuvo una masa gelatinosa, a la cual le dio el nombre de "algina", derivado de alga. ${ }^{1}$ En los últimos años estos materiales han evolucionado considerablemente, siendo utilizados para la reproducción exacta de dientes y estructuras orales mediante la toma de impresiones, haciéndose necesaria la utilización de materiales con distorsión mínima o nula para asegurar así el éxito del tratamiento a realizar. ${ }^{2,3}$ Nicolls define distorsión como el movimiento relativo de un sólo punto o un grupo de puntos lejos de su posición de referencia original especificada. ${ }^{4}$ Él establece que desde el punto de vista matemático, el movimiento tiene tres posibles desplazamientos lineales y tres desplazamientos rotacionales en los tres ejes del espacio (X, Y, y Z). ${ }^{5,6}$

Por tanto el paso clave para disminuir esta distorsión en los modelos de trabajo para todo procedimiento de rehabilitación oral es la impresión teniendo en cuenta la importancia de controlar al máximo las condiciones del ambiente de trabajo y la correcta manipulación del material. ${ }^{7}$

\section{4- MATERIALES Y MÉTODOS}

El presente estudio fue experimental, efectuado en la facultad de Odontología de la UNAN-LEÓN. La unidad de estudio fue el modelo de piedra dental tipo IV y la población la constituyeron 20 modelos de piedra dental tipo IV, 5 por cada hidrocoloide irreversible. Para la obtención de este se diseñó un Modelo Maestro fabricado en aluminio, todas las superficies fueron pulidas para minimizar la retención a consecuencia de las rugosidades.

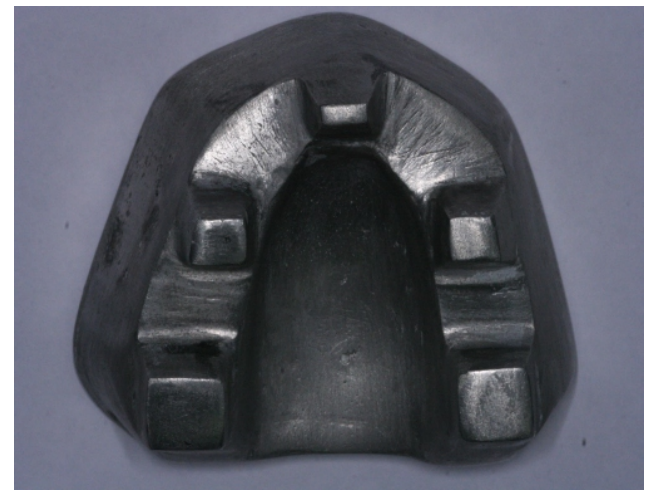

FIG.1 Modelo Maestro

Se utilizó un pequeño pilar de $9 \mathrm{~mm}$ de altura para el incisivo central, usando un punto de referencia incisal a nivel de sus vértices. Dos pequeños pilares se colocaron a cada lado del arco dental en la región de las primeras premolares superiores con una altura de $7 \mathrm{~mm}$ para cada una de ellas y en la región de las segundas molares superiores con una altura de $6 \mathrm{~mm}$ para dichos pilares. La distancia entre el pilar que simula el incisivo central superior al primer premolar es de $18 \mathrm{~mm}$ y del primer premolar a la segunda molar es de $13 \mathrm{~mm}$ por hemiarcada. Se diseñó cada diente en forma cuadrangular para disminuir la retención del material durante la toma de impresión. Se establecieron un total de 7 puntos de medida sobre el modelo estándar de referencia y los modelos en piedra dental tipo IV, los cuales fueron: 
- DeA-B, de un punto disto-vestibular del pilar 1 hacia un punto mesio-palatino del pilar 2.

- DeA-C, de un punto a disto-vestibular del pilar 1 hacia un punto mesio-vestibular del pilar 3

- DeA-D, de un punto disto-vestibular del pilar 1 hacia un punto mesio-vestibular del pilar 4.

- DeA-E, de un punto disto-vestibular del pilar 1 hacia un punto mesio-vestibular del pilar 5.

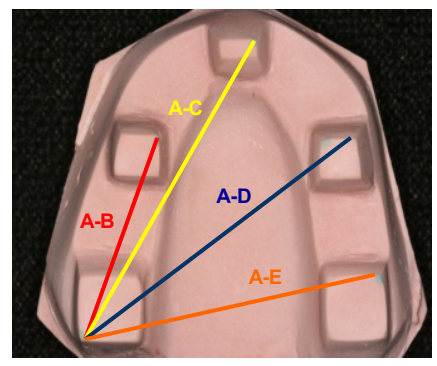

FIG.2 Puntos de medidas

- De A-A ${ }^{1}$, medición vestíbulo-palatino en la misma pieza dental (segunda molar superior derecha).

- De B-B ${ }^{1}$, medición vestíbulo- palatino en la misma pieza dental (primer premolar superior derecho).

- De C-C $\mathrm{C}^{1}$, medición vestíbulo- palatino en la misma pieza dental (incisivo).

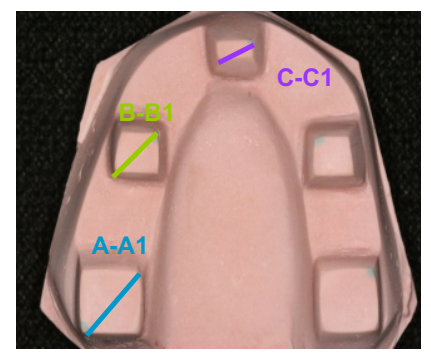

FIG.3 Puntos de medidas

Se fabricó un instrumento especial para la estandarización de las impresiones, el cual constaba de una base inferiory una base superior de acrílico cuyas medidas son de $1.6 \mathrm{~cm}, 16 \mathrm{~cm}$ y $16 \mathrm{~cm}$. La base inferior constaba de 3 barras cilíndricas fijas posicionadas transversalmente formando un triángulo isósceles entre ellas y en el centro se sujetó el modelo maestro. En la base superior se hicieron tres perforaciones a través de las cuales pasaban las tres barras cilíndricas para permitir el desplazamiento en un solo eje de entrada y salida. En el centro se sujetó la cubeta de impresión rim-lock, tamaño L.

FIG. 4 Instrumento de Estandarización de Impresiones.

A) Base superior, B) Base inferior, C) Modelo maestros,

D) Cubeta Rim-lock, E) Barras cilíndricas F) Topes verticales.

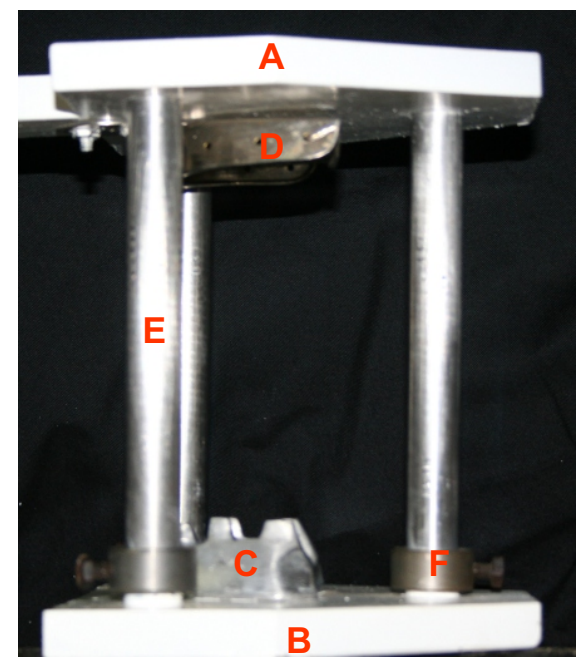


Se tomaron 5 impresiones por cada hidrocoloide irreversible. Un examinador capacitado ${ }^{1}$ se encargó de mezclar el alginato según las indicaciones dadas por cada fabricante, luego se vertió el material en la cubeta de impresión y el examinador ${ }^{2}$ tomó la impresión, mientras que el examinador ${ }^{3}$ manipuló el yeso piedra tipo IV para vaciarlo y sacar los modelos de estudio, todo se realizó bajo la temperatura adecuada mediante el uso de termómetros y termostatos con previa calibración a $21^{\circ} \mathrm{C}$ (temperatura ambiente). Las muestras fueron tomadas a horas determinadas de 8:00 a 9:00 a.m.

Se tomó la impresión del modelo con un tiempo de permanencia de acuerdo a la especificación del fabricante, posteriormente se lavó la impresión para disminuir la tensión superficial y se secó con aire comprimido durante 10 segundos. El vaciado se realizó entre los 10 minutos después de la toma de impresión con piedra dental tipo IV (Silky- Rock, Violet Whip-Mix ${ }^{\circledR}$ Corporation, Louisville, Kentucky) en una proporción de 100 gr. de piedra por 23 ml de agua destilada.

Se mezcló de forma manual por 30 segundos y posteriormente se mezcló mecánicamente en la mezcladora al vacío marca Buffalo por 50 segundos, se vibró la cubeta en un vibrador marca Buffalo Dental Mfg. Co. Inc. Syosset, NY. Finalmente se colocó la cubeta en una tabla ranurada y se esperó un periodo de 45 a 60 minutos para garantizar el fraguado de la piedra dental.

Al cumplirse el tiempo, los modelos se retiraron de la cubeta de impresión, se recortaron en una recortadora marca whip mix modelo 5KH39QN9741HX, y se alisaron con lija de agua 365.

A cada grupo de modelo se le dio un código para evitar cualquier confusión entre una marca y otra. Se utilizó una máquina de medición por coordenadas tridimensionales (CMM, Mitutoyo BH-305, Japón) con un programa de cómputo Geopack 500 versión 2.0 ubicada en el laboratorio del Centro de Formación de Formadores y de Personal Técnico para el Desarrollo Industrial de Centroamérica (CEFOF) en el Laboratorio Metal Mecánica, Alajuela, Costa Rica con certificación ISO 9002. Un vástago con una esfera de $1 \mathrm{~mm}$ en su extremo fue el sensor de la máquina tridimensional.

Esta máquina tiene la capacidad de medir cambios en la posición, distancias y sacar centroides en el espacio en las tres coordenadas (X, Y, Z) con grado significativo de $0.0001 \mathrm{~mm}$ dando un resultado instantáneo.
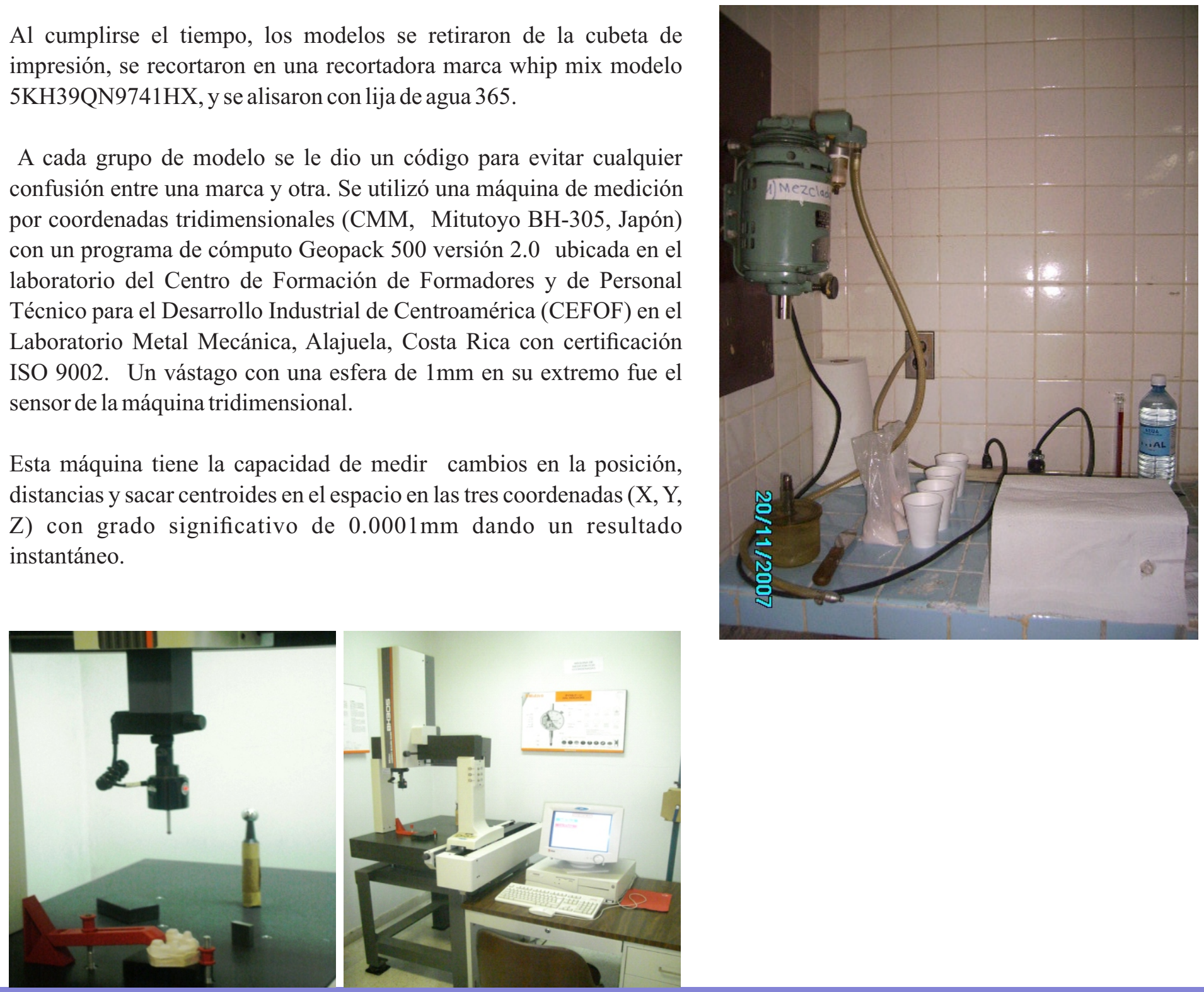
La selección de los grupos y modelos fue aleatoria para su medición. Se realizó la medición del modelo maestro por ser el patrón y consecutivamente los grupos de piedra dental de acuerdo a su aleatorización. El orden que se obtuvo fue Jeltrate, Vival NF, Rite Dent y Max Print Cyan. A los 7 puntos de medidas (A-B, A-C, A-D, A-E, A-A ${ }^{1}, B_{-} B^{1}, C_{-} C^{1}$ ), se le realizaron tres mediciones por cada distancia y se sacó un promedio para estandarizar los datos y minimizar sesgos, obteniendo 420 mediciones en 20 modelos de estudio y 35 en el modelo maestro

logrando un total de 455 mediciones. Las mediciones realizadas fueron de tipo lineal. Los datos previamente codificados se tabularon en una base de datos relacional creada por el paquete estadístico SPSS versión 12.0.

El análisis estadístico se realizó mediante el uso de las estadísticas descriptivas ANOVAy test de Tukey.

\section{5- RESULTADOS}

Tabla I. Valores mínimos, máximos, media y desviación estándar de los cuatro hidrocoloides irreversibles y modelo maestro en los puntos de medida de $A-B, A-C, A-D, A-E$.

\begin{tabular}{cccccc}
\hline Puntos de medida & $\mathrm{N}$ & $\begin{array}{c}\text { Mínimo } \\
(\mathrm{mm})\end{array}$ & $\begin{array}{c}\text { Máximo } \\
(\mathrm{mm})\end{array}$ & $\begin{array}{c}\text { Media } \\
(\mathrm{mm})\end{array}$ & $\begin{array}{c}\text { Desviación Std. } \\
(\mathrm{mm})\end{array}$ \\
\hline De A-B & $\mathbf{2 5}$ & $\mathbf{3 0 . 5 5}$ & $\mathbf{3 1 . 8 1}$ & $\mathbf{3 1 . 3 3 0 2}$ & $\mathbf{0 . 3 1 0 7 8}$ \\
De A-C & 25 & 50.25 & 51.67 & 50.7842 & 0.42480 \\
De A-D & 25 & 49.38 & 51.34 & 49.9485 & 0.54464 \\
De A-E & $\mathbf{2 5}$ & $\mathbf{5 1 . 6 1}$ & $\mathbf{5 3 . 6 5}$ & $\mathbf{5 2 . 8 3 6 3}$ & $\mathbf{0 . 5 1 2 6 5}$ \\
\hline
\end{tabular}

El punto de medida que obtuvo un mayor valor de su media fue $(A-E)$ con $52.8363 \mathrm{~mm}$ el de menor valor $(A-B)$ con $31.3302 \mathrm{~mm}$, la mayor desviación estándar fue en el punto de medida (A-D) con $0.54464 \mathrm{~mm}$ y el de menor fue (A-B) con $0.31078 \mathrm{~mm}$.

Tabla II. Análisis de variancia de los puntos de medida de AB, A-C, A-D, A-E.

\begin{tabular}{cccccc}
\hline Puntos de medida & Suma de cuadrados & Diferencia & $\begin{array}{r}\text { Media } \\
(\mathrm{mm})\end{array}$ & $\begin{array}{c}\text { F } \\
(\mathrm{mm})\end{array}$ & $\begin{array}{c}\text { Sig. } \\
(\mathrm{mm})\end{array}$ \\
\hline De A-B & 0.313 & 4 & 0.078 & 0.780 & 0.551 \\
De A-C & 1.520 & 4 & 0.380 & 2.705 & 0.060 \\
De A-D & 3.371 & 4 & 0.843 & 4.497 & 0.009 \\
De A-E & 1.057 & 4 & 0.264 & 1.007 & 0.427 \\
\hline
\end{tabular}

El punto de medida AD fue el único con valor estadísticamente significativo con $0.009 \mathrm{~mm}$ 
Tabla III. Análisis Tukey HSD (diferencia verdaderamente significativa) del punto de medida A-D, de los cuatro tratamientos en comparación con el modelo maestro.

\begin{tabular}{|c|c|c|}
\hline \multicolumn{2}{|c|}{ Tratamiento } & Significancia \\
\hline & Jeltrate & $0.165 \mathrm{~mm}$ \\
\hline Modelo & Vival NF & $0.008 \mathrm{~mm}$ \\
\hline \multirow[t]{2}{*}{ Maestro } & Rite Dent & $0.052 \mathrm{~mm}$ \\
\hline & Print Gel & $0.714 \mathrm{~mm}$ \\
\hline
\end{tabular}

Únicamente en el punto de medida A-D se encontró diferencia estadísticamente significativa de $0.008 \mathrm{~mm}$ en el material Vival NF.

Tabla IV. Valores mínimos, máximos, media y desviación estándar de los cuatro hidrocoloides irreversibles y modelo maestro en los puntos de medida de A-A1, B-B1, C-C1.

\begin{tabular}{crrrrc}
\hline Puntos de medida & $\mathrm{N}$ & $\begin{array}{r}\text { Mínimo } \\
(\mathrm{mm})\end{array}$ & $\begin{array}{r}\text { Máximo } \\
(\mathrm{mm})\end{array}$ & $\begin{array}{r}\text { Media } \\
(\mathrm{mm})\end{array}$ & $\begin{array}{r}\text { Desviación Std. } \\
(\mathrm{mm})\end{array}$ \\
\hline & & & & & \\
De A-A ${ }^{1}$ & 25 & 11.82 & 13.34 & 12.4444 & 0.28614 \\
De B-B 1 & 25 & 8.07 & 9.40 & 8.5422 & 0.30873 \\
De C-C & 25 & 5.64 & 6.78 & 6.4090 & 0.28277 \\
\hline
\end{tabular}

El punto de medida que obtuvo un mayor valor de su media fue (A-A1) con $12.4444 \mathrm{~mm}$, el de menor valor (C-C1) con $6.4090 \mathrm{~mm}$, la mayor desviación estándar se obtuvo en el punto de medida B-B1 con $0.30873 \mathrm{~mm}$ y la menor en (C-C1) con $0.28277 \mathrm{~mm}$.

Tabla V. Análisis de variancia de los puntos de medida de A-A1, B-B1, C-C1

\begin{tabular}{cccccc}
\hline $\begin{array}{c}\text { Puntos de } \\
\text { medida }\end{array}$ & $\begin{array}{c}\text { Suma de } \\
\text { cuadrado }\end{array}$ & Diferencia & $\begin{array}{c}\text { Media } \\
(\mathrm{mm})\end{array}$ & $\begin{array}{c}\mathrm{F} \\
(\mathrm{mm})\end{array}$ & $\begin{array}{c}\text { Sig. } \\
(\mathrm{mm})\end{array}$ \\
\hline & & & & & \\
De A-A & 0.698 & 4 & 0.174 & 2.752 & 0.057 \\
De B-B & 0.367 & 4 & 0.092 & 0.957 & 0.453 \\
De C-C & 0.442 & 4 & 0.111 & 1.497 & 0.241 \\
\hline
\end{tabular}

No se encontraron diferencias significativas en ninguno de los puntos de medida. 


\section{6- CONCLUSIONES}

- No se encontró diferencia estadísticamente significativa en los puntos de A-B, A-C, A-E a excepción del punto A-D en el hidrocoloide irreversible marca Vival NF.+

- En los puntos de medida A-A ${ }^{1}, B_{-} B^{1}, C-C^{1}$ no se encontró significancia estadística para ninguno de los materiales usados (Jeltrate, Max Print Cyant, Vival NF y Rite Dent).

\section{7- REFERENCIAS BIBLIOGRÁFICAS}

1- Ralph W. Phillips. La ciencia de los materiales dentales. Tercera edición en español, 1993. Editorial Interamericana, S.A. Pág. 125-135.

2- $\quad$ Robert G. Craig; William J. O'brien; John M. Powers. Materiales dentales. Sexta edición, 1996. editorial Harcourt Brace. Pág. 136-155.

3- Robert G. Craing; Marrcus L. Materiales de odontología restauradora. Decima edición. 1998. editorial Harcourt Brace. Pág. 281-300.

4- J.I.Nicholls. The measurement of distortion. Theoretical considerations_-J. Prosthet Dent. 1977; 37 (5): 578-586.

5- J.I.Nicholls The measurement of distortion: concluding remarks. J Prosthet Dent 1980; Feb. volume 43 (2): $218-223$.

6- J.I.Nicholls The measurement of distortion: mathematical considerations. J Prosthet Dent. 1978; Mar; 39 ( 3): 339 343.

7- $\quad$ B.A Linke,J.I.Nicholls and R.R Faucher. Distortion analysis cast made from impression materials. J Prosthet Dent. Dec. $1985 ; 54 ;(6) ; 794-802$.

8- $\quad$ Lucas Da Fonseca Roberti Garcia, Simonides Consani, Ingrid Machado de Andrade,y Fernanda de Carvalho Panzeri Pires- de- Souza. Análise crítica dos fatores que influenciam a precisao de moldagens com elastómeros. 2006 pág. (387- 391).

9- $\quad$ Steas. A new method for making casts from irreversible hidrocolloid impressions. March 1991, volume 65, number 3 pág. (454-456).

10- $\quad$ Ayne W. Daniel. Bioestadística, Base para el análisis de las ciencias de la salud. 4ª Edición. 2002, Editorial Limusa, S.A. de C.V. Grupo Noriega editores.

11-Gennari Filho, Humberto*; Vedovatto, Eduardo**; Quinelli Mazaro, José Vitor**; Coelo Goiato, Marcelo*; Dos Santos, Paulo Enrique. Influence of finger-smoothed moistening of irreversible hidrocolloid on dimension and surface changes of molds. RCOE v.12 n.1-2 Madrid ene.-jun. 2007. 
12-- Wayne W. Daniel. Bases para el análisis de las ciencias de la salud. Bioestadística $4^{a}$ edición. Editorial Limusa. 2002,

13- Matthias KERN, Reinhold M. and Jorg R. Three-dimensional investigation of the accuracy of impression materials after disinfection.

14- Propiedades físico-químicas, utilidad y proceso productivo de los Alginatos www.monografias.com/trabajos12/alginato/alginato.shtml.

15- Indiana López Castillo. Comparación con la exactitud de dos materiales de impresión en la elaboración de modelos de trabajo para prótesis fija. Universidad de Costa Rica 2008.

16- Bello López René, Camacho Arenas Alejandra, Galván Bautista Naxhllely, García Sánchez Ana, Sánchez Ruiz Karla. Distorsión de los modelos tomados con alginato $<$ Tropicalgin $>$, alginato siliconizado $<$ Max print $>$, y silicona $<$ Zeta Plus $>$ en dientes naturales montados en caja di lock". 